\title{
Painéis aglomerados produzidos com seis espécies de madeiras tropicais da Amazônia
}

\section{Particleboard manufactured from six species of Amazon tropical timbers}

\author{
Setsuo Iwakiri ${ }^{*}$, Rosilani Trianoskil', Claudete Catanhede do Nascimento², Claudio Gumane Francisco Juizo³, \\ Elaine Cristina Lengowski³, Géssica Katalyne Bilcati ${ }^{3}$ e Thais Gonçalves ${ }^{3}$
}

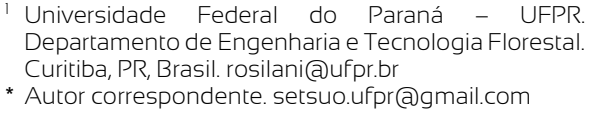

* Autor correspondente. setsuo.ufpr@gmail.com

\section{RESUMO}

Esta pesquisa teve como objetivo avaliar a qualidade de painéis aglomerados produzidos com seis espécies de madeiras tropicais da Amazônia: Byrsonima crispa, Eschweilera coriacea, Eschweilera odora, Manilkara amažonica, Pouteria guianensis e Swartzia recurva. Foram produzidos painéis experimentais com densidade nominal de $0,75 \mathrm{~g} / \mathrm{cm}^{3}$, utilizando a resina uréia-formaldeído na proporção de $8 \%$ de sólidos - base peso seco das partículas. Os painéis foram prensados com pressão específica de $4,0 \mathrm{MPa}$, temperatura de $160^{\circ} \mathrm{C}$ e tempo de prensagem de oito minutos. Os painéis foram avaliados com base nas seguintes propriedades: razão de compactação, absorção de água e inchamento em espessura 2 horas e 24 horas, módulos de elasticidade e de ruptura, tração perpendicular, resistência ao arrancamento de parafusos na superfície e topo. A baixa razão de compactação dos painéis produzidos comas espécies tropicais não afetou de forma significativa os resultados de suas propriedades físicas e mecânicas. Avaliações comparativas dos resultados obtidos com os requisitos da norma EN 312 (2003) e espécie referencial - Pinus taeda, demonstraram que as espécies Byrsonima crispa, Eschweilera coriacea, Eschweilera odora e Pouteria guianensis apresentam grande potencial para produção de painéis aglomerados.

PALAVRAS-CHAVE: partículas de madeira, razão de compactação, ureia-formaldeído.

\begin{abstract}
This research aimed to evaluate the quality of particleboard produced with six Amazonian tropical woods: Byrsonima crispa, Eschweilera coriacea, Eschweilera odora, Manilkara amazonica, Pouteria guianensis and Swartzia recurva. Experimental panels were produced with nominal density of $0,75 \mathrm{~g} / \mathrm{cm}^{3}$, using the urea-formaldehyde resin in a proportion of $8 \%$ solids based on dry weight of the particles. The panels were pressed with a specific pressure of $4,0 \mathrm{MPa}$, temperature of $160^{\circ} \mathrm{C}$ and pressing time of eight minutes. The panels were evaluated based on the following properties: compression ratio, water absorption and thickness swelling for 2 hours and 24 hours, modulus of elasticity and modulus of rupture, tension perpendicular, screws pullout resistance on the surface and top. Low compression ratio of panels produced with tropical wood species did not affect significantly the results of their physical and mechanical properties. Comparative evaluation of the results obtained with the requirements of EN 312 (2003) and reference species - Pinus taeda, showed that Byrsonima crispa, Eschweilera coriacea, Eschweilera odora and Pouteria guianensis have great potential for the production of particleboard.
\end{abstract}

KEYWORDS: wood particles, compaction ratio, urea-formaldehyde. 


\section{INTRODUÇÃO}

As indústrias de painéis aglomerados no Brasil estão instaladas nas regiões sul e sudeste, para atender à demanda dos principais polos moveleiros localizados nos Estados de São Paulo, Minas Gerais, Paraná, Santa Catarina e Rio Grande do Sul. As florestas plantadas de Pinus e Eucalipto são as bases de suprimento de madeira para as indústrias de painéis aglomerados. Embora as regiões norte e centrooeste do país tenham disponíveis extensas áreas de florestas tropicais nativas, poucos estudos têm sido realizados sobre a viabilidade de utilização de madeiras tropicais para produção de painéis aglomerados. $\mathrm{O}$ aproveitamento de resíduos provenientes das serrarias e laminadoras, além de espécies pouco conhecidas comercialmente, pode ser uma alternativa interessante para instalação de pequenas fábricas de painéis aglomerados na região amazônica e, por conseguinte, abrir perspectivas de instalação de polos moveleiros na região, reduzindo as dependências de importação de móveis de regiões sul e sudeste do país a custos elevados (Iwakiri, Vianez, Weber, Trianoski e Almeida 2012).

Uma intensificação no uso da madeira como matériaprima para fins industriais ou construtivos só pode ocorrer a partir do conhecimento adequado de suas propriedades, sejam elas físicas, químicas ou mecânicas. A madeira, por ser elemento orgânico, heterogêneo e composto basicamente de celulose, hemicelulose, lignina e extrativos, apresenta enorme versatilidade de usos para obtenção de uma série de produtos (Gonçalves, Oliveira, Lucia e Sartório, 2009).

As propriedades anatômicas, físicas e químicas das diferentes espécies florestais são parâmetros importantes na fabricação de produtos reconstituídos de madeira. Segundo Marra (1992), as propriedades da madeira possuem forte influência na formação da ligação adesiva e, geralmente, as madeiras de folhosas apresentam mais dificuldades para colagem do que as de coníferas devido a sua composição química diferenciada.

A escolha de espécies de madeira para produção de painéis aglomerados deve ser baseada em alguns parâmetros como a densidade, $\mathrm{pH}$ e extrativos (Moslemi,
1974, Maloney, 1993, Tsoumis, 1991). A densidade da madeira é um dos requisitos básicos na escolha de espécies para produção de aglomerados, devido a sua influência na razão de compactação, que é a relação entre a densidade do painel e a densidade da madeira. Segundo os autores, a razão de compactação deve ser de no mínimo 1,3 para assegurar uma área de contato satisfatória entre as partículas e densificação suficiente para a formação do painel. Kelly (1977), afirma que, para painéis de mesma densidade, produzidos com madeira de baixa densidade, as suas propriedades mecânicas serão superiores, entretanto, a sua estabilidade dimensional será inferior em comparação aos painéis produzidos com madeira de alta densidade. Segundo o autor, nos painéis com maior razão de compactação, há maior quantidade de partículas de madeira e, consequentemente, ocorrerá maior densificação do painel, resultando em maior inchamento higroscópico da madeira e liberação de maiores tensões de compressão geradas durante o processo de prensagem à alta temperatura. Maloney (1993) afirma que as espécies com densidade de até $0,55 \mathrm{~g} / \mathrm{cm}^{3}$ são as mais adequadas para produção de painéis de partículas de madeira, por atingirem uma razão de compactação entre 1,3 e 1,6. Numa pesquisa conduzida por Vital, Lehmann, e Boone (1974) sobre painéis aglomerados produzidos com Virola spp., uma espécie de madeira tropical da Amazônia, com massa específica aparente de $0,43 \mathrm{~g} / \mathrm{cm}^{3}$, o aumento na razão de compactação dos painéis de 1,2 (baixa) a 1,6 (alta), resultaram em aumentos significativos nos resultados de tração perpendicular e flexão estática (MOE e MOR).

Com relação ao $\mathrm{pH}$ e extrativos da madeira, Marra (1992) afirma que estes parâmetros podem influenciar diretamente na cura da resina e, consequentemente, na qualidade dos painéis produzidos. $\mathrm{O} \mathrm{pH}$ da madeira pode variar entre 3,0 a 5,5 e de acordo com Kelly (1977), madeiras com pH muito ácido podem causar a pré-cura da resina uréia-formaldeído durante a fase de fechamento da prensa, prejudicando o grau de adesão entre as partículas e reduzindo os valores das propriedades mecânicas dos painéis. Por outro lado, madeiras que apresentam $\mathrm{pH}$ pouco ácido requerem quantidade um pouco maior de 
catalisador para acelerar a cura da resina uréia-formaldeído. Quanto a influência dos extrativos na polimerização e cura do adesivo, Marra (1992) afirma que madeiras com elevados teores de extrativos apresentam dificuldades de colagem resultando em baixa resistência da ligação adesiva entre as partículas.

\section{OBJETIVOS}

Este trabalho teve como objetivo avaliar a qualidade de painéis aglomerados produzidos com seis espécies de madeiras tropicais da Amazônia, com base na análise comparativa dos resultados de suas propriedades físicas e mecânicas com painéis de Pinus taeda e requisitos da norma EN 312:2002 (European Standard [EN], 2003).

\section{MATERIAIS E MÉTODOS}

\section{Materiais}

As seis espécies de madeiras tropicais estudadas neste trabalho foram: Byrsonima crispa, Eschweilera coriacea, Eschweilera odora, Manilkara amazonica, Pouteria guianensis e Swartzia recurva, provenientes da Estação Experimental ZF2 do Instituto Nacional de Pesquisas da Amazônia, localizada no Município de Manaus, Estado do Amazonas. Como referência comparativa foram utilizadas partículas de madeira de Pinus taeda coletadas numa indústria de aglomerados localizada no município de Araucária, Estado do Paraná, tendo em vista ser a espécie mais empregada para produção de painéis aglomerados no Brasil. Para a colagem, foi utilizada a resina ureia-formaldeído com teor de sólidos de $65 \%$, catalisador a base de sulfato de amônia e emulsão de parafina.

\section{Metodologia de pesquisa}

As amostras de madeiras foram obtidas na forma de pranchas a partir de três árvores coletadas aleatoriamente na floresta e posteriormente transformadas em partículas de madeira num picador de disco com as seguintes dimensões nominais: comprimento de $25 \mathrm{~mm}$, espessura de $0,7 \mathrm{~mm}$ e largura variável. Após a secagem ao teor de umidade médio de $3 \%$, as partículas foram reprocessadas no moinho de martelo para redução nas dimensões e classificadas em peneira de malha 0,6 para retirada de "finos".

A massa específica básica da madeira foi determinada de acordo com procedimentos recomendados pela Norma COPANT 461:1972 (Comissão Panamericana de Normas Técnicas [COPANT], 1972). Já com relação às propriedades químicas, as análises realizadas foram extrativos totais (Technical Association of the Pulp and Paper Industry [TAPPI], 1997) e pH (TAPPI, 2002).

Foram produzidos em laboratório três painéis aglomerados para cada espécie, num total de 21 painéis, com as dimensões de $50 \mathrm{~cm} \times 38 \mathrm{~cm} \times 1,3 \mathrm{~cm}$ e densidade nominal de $0,75 \mathrm{~g} / \mathrm{cm}^{3}$, conforme plano experimental apresentado na tabela 1.

TABELA 1. Plano experimental.

\begin{tabular}{cl}
\hline Tratamento & \multicolumn{1}{c}{ Espécie } \\
\hline 1 & Byrsonima crispa \\
2 & Eschweilera coriacea \\
3 & Eschweilera odora \\
4 & Manilkara amazonica \\
5 & Pouteria guianensis \\
6 & Swartzia recurva \\
7 & Pinus taeda \\
\hline
\end{tabular}

A resina ureia-formaldeído catalisada com sulfato de amônia foi aplicada sobre as partículas em quantidade de $8 \%$ de sólidos em relação ao peso seco das partículas. Foi aplicada também 1\% de emulsão de parafina com finalidade de reduzir a absorção de água. Para a formação do colchão de partículas foi utilizada uma caixa formadora vazada com dimensões laterais de $50 \mathrm{~cm} \times 38 \mathrm{~cm}$ e uma barra de aço com espessura de $1,3 \mathrm{~cm}$ como limitador de espessura do painel. Os painéis foram prensados à temperatura de 160 ${ }^{\circ} \mathrm{C}$, pressão específica de 4,0 $\mathrm{MPa}$ e tempo de prensagem de oito minutos.

Após a prensagem, os painéis foram esquadrejados e acondicionados em câmara climática com temperatura de $20{ }^{\circ} \mathrm{C} \pm 2{ }^{\circ} \mathrm{C}$ e umidade relativa de $65 \% \pm 3 \%$, até sua estabilização ao teor de umidade médio de $12 \%$. 
Para avaliação das propriedades físico-mecânicas foram retirados de cada painel cinco corpos-de-prova para ensaios de massa específica aparente, quatro para flexão estática, cinco para tração perpendicular (ligação interna), cinco para absorção de água e inchamento em espessura após duas e 24 horas de imersão em água, três para arrancamento de parafusos na superfície e três no topo. Os ensaios foram realizados de acordo com os procedimentos descritos na Norma Europeia EN 323:2003, EN 310:2003, EN 319:2003, EN 317:2003 e NBR 14810-3:2006 (ES, 2002d; 2002a; 2002c; 2002b; Associação Brasileira de Normas Técnicas [ABNT], 2006b), respectivamente.

Os resultados obtidos foram submetidos a analise estatística na ferramenta Statgraphics Centurion XV-II e os testes foram Grubbs, Skewness, Kurtosis, Bartlett, Análise de Variância. Uma vez rejeitada a hipótese nula, afirmando uma fonte única dos dados, foram realizados testes de medias de Tukey (95\% de probabilidade).

\section{Resultados}

\section{Propriedades da madeira}

$\mathrm{Na}$ tabela 2 estão apresentados os valores médios de massa específica básica, teor de extrativos totais e $\mathrm{pH}$ da madeira para as seis espécies estudadas.

A Eschweilera odora foi a espécie que apresentou menor massa específica básica com $0,637 \mathrm{~g} / \mathrm{cm}^{3}$ e a Manilkara amaẑñica a que apresentou maior valor médio com 0,841 $\mathrm{g} / \mathrm{cm}^{3}$, sendo consideradas madeiras de média a alta massa específica básica.

Os valores médios de teor de extrativos totais variaram na faixa de 3,16\% para Pouteria guianensis e 9,24\% para Manilkara amazônica. Com exceção das madeiras de Pouteria guianensis e Byrsonima crispa, as demais espécies tropicais apresentaram valores de extrativos totais bem acima do valor médio obtido para Pinus taeda de 3,37\%. Com relação ao $\mathrm{pH}$, os valores médios variaram de 3,99 para Manilkara amazônica e de 6,16 para Pouteria guianensis.

\section{Propriedades físicas dos painéis}

$\mathrm{Na}$ tabela 3 estão apresentados os valores médios de massa específica, razão de compactação, absorção de água e inchamento em espessura após 2 horas e 24 horas de imersão em água dos painéis.

Os valores médios de massa específica dos painéis variaram de $0,697 \mathrm{~g} / \mathrm{cm}^{3}$ para painéis de Pinus taeda a 0,749 $\mathrm{g} / \mathrm{cm}^{3}$ para painéis de Byrsonima crispa. A razão de compactação dos painéis variou de 0,78 para painéis de Swartzia recurva e 1,59 para painéis de Pinus taeda. Tanto para a massa específica, quanto para a razão de compactação, foram constatadas diferenças estatisticamente significativas entre as médias obtidas para as diferentes espécies.

TABELA 2. Propriedades da madeira.

\begin{tabular}{lccc}
\hline Espécie & $M E_{\text {básica }}\left(\mathrm{g} / \mathrm{cm}^{3}\right)$ & Extrativos totais $(\%)$ & $p H$ \\
\hline Byrsonima crispa & $0,689(15,09)$ & $3,19(3,07)$ & $5,35(5,14)$ \\
Eschweilera coriacea & $0,748(16,45)$ & $7,79(4,26)$ & $4,91(2,75)$ \\
Eschweilera odora & $0,637(9,74)$ & $7,68(9,82)$ & $5,00(4,61)$ \\
Manilkara amazonica & $0,841(9,92)$ & $9,24(5,82)$ & $3,99(6,90)$ \\
Pouteria guianensis & $0,738(4,24)$ & $3,16(7,36)$ & $6,16(4,88)$ \\
Swartzia recurva & $0,749(5,33)$ & $6,53(5,29)$ & $5,18(3,80)$ \\
Pinus taeda & 0,450 & $3,37(6,12)$ & $4,58(0,88)$ \\
\hline
\end{tabular}

ME: Massa específica; Valores entre parênteses referem-se ao coeficiente de variação. 
TABELA 3. Resultados médios das propriedades físicas dos painéis.

\begin{tabular}{|c|c|c|c|c|c|c|}
\hline Tratamento & $\begin{array}{l}M E_{12 \%} \\
\left(\mathrm{~g} / \mathrm{cm}^{3}\right)\end{array}$ & $R C$ & $\begin{array}{l}\text { AA } 2 h \\
\text { (\%) }\end{array}$ & $\begin{array}{l}\text { AA } 24 h \\
\text { (\%) }\end{array}$ & $\begin{array}{l}\text { IE } 2 h \\
\text { (\%) }\end{array}$ & $\begin{array}{l}\text { IE } 24 h \\
\text { (\%) }\end{array}$ \\
\hline Byrsonima crispa & $\begin{array}{l}0,749 \mathrm{a} \\
(6,43)\end{array}$ & $\begin{array}{l}0,88 \mathrm{c} \\
(5,75)\end{array}$ & $\begin{array}{l}23,27 \mathrm{~b} \\
(33,32)\end{array}$ & $\begin{array}{l}47,70 \text { b } \\
(21,89)\end{array}$ & $\begin{array}{l}7,78 \mathrm{~b} \\
(18,05)\end{array}$ & $\begin{array}{l}16,66 \mathrm{bc} \\
(19,17)\end{array}$ \\
\hline Eschweilera coriacea & $\begin{array}{l}0,730 \text { a } \\
(4,14)\end{array}$ & $\begin{array}{l}0,96 \mathrm{~b} \\
(4,81)\end{array}$ & $\begin{array}{l}7,34 \mathrm{e} \\
(25,12)\end{array}$ & $\begin{array}{l}22,52 \mathrm{~d} \\
(15,97)\end{array}$ & $\begin{array}{l}2,05 \mathrm{c} \\
(30,77)\end{array}$ & $\begin{array}{l}8,28 \mathrm{~cd} \\
(14,28)\end{array}$ \\
\hline Eschweilera odora & $\begin{array}{l}0,730 \mathrm{a} \\
(5,61)\end{array}$ & $\begin{array}{l}0,82 d \\
(6,22)\end{array}$ & $\begin{array}{l}15,22 \mathrm{c} \\
(18,32)\end{array}$ & $\begin{array}{l}39,53 \mathrm{c} \\
(17,65)\end{array}$ & $\begin{array}{l}3,65 c \\
(49,60)\end{array}$ & $\begin{array}{l}13,38 \mathrm{c} \\
(18,53)\end{array}$ \\
\hline Manilkara amazonica & $\begin{array}{l}0,741 \text { a } \\
(5,95)\end{array}$ & $\begin{array}{l}0,90 \mathrm{c} \\
(4,89)\end{array}$ & $\begin{array}{l}10,81 \mathrm{~d} \\
(30,02)\end{array}$ & $\begin{array}{l}23,79 \mathrm{~d} \\
(20,52)\end{array}$ & $\begin{array}{l}2,46 \mathrm{c} \\
(37,72)\end{array}$ & $\begin{array}{l}6,89 \mathrm{~d} \\
(21,59)\end{array}$ \\
\hline Pouteria guianensis & $\begin{array}{l}0,738 \text { a } \\
(5,38)\end{array}$ & $\begin{array}{l}0,99 \mathrm{~b} \\
(5,44)\end{array}$ & $\begin{array}{l}20,18 \text { b } \\
(31,23)\end{array}$ & $\begin{array}{l}40,35 \text { bc } \\
(16,86)\end{array}$ & $\begin{array}{l}4,09 \mathrm{c} \\
(40,93)\end{array}$ & $\begin{array}{l}12,04 \mathrm{c} \\
(18,70)\end{array}$ \\
\hline Swartzia recurva & $\begin{array}{l}0,741 \text { a } \\
(6,81)\end{array}$ & $\begin{array}{l}0,78 \mathrm{~d} \\
(7,29)\end{array}$ & $\begin{array}{l}22,35 \text { b } \\
(29,10)\end{array}$ & $\begin{array}{l}44,19 b \\
(16,27)\end{array}$ & $\begin{array}{l}7,72 \mathrm{~b} \\
(22,26)\end{array}$ & $\begin{array}{l}18,74 \mathrm{~b} \\
(13,49)\end{array}$ \\
\hline Pinus taeda & $\begin{array}{l}0,697 \text { b } \\
(5,35)\end{array}$ & $\begin{array}{l}1,59 a \\
(5,74)\end{array}$ & $\begin{array}{l}48,18 a \\
(17,94)\end{array}$ & $\begin{array}{l}85,18 \text { a } \\
(6,01)\end{array}$ & $\begin{array}{l}15,49 \text { a } \\
(23,45)\end{array}$ & $\begin{array}{l}35,23 a \\
(12,02)\end{array}$ \\
\hline
\end{tabular}

ME: Massa específica; RC: razão de compactação; AA: Absorção de água; IE: Inchamento em espessura; médias seguidas da mesma letra na mesma coluna são estatisticamente iguais pelo teste de Tukey a 95\% de probabilidade. Valores entre parêntese indicam o coeficiente de variação. Resultados de AA e IE ajustados pela análise de covariância para massa especifica aparente de $0,745 \mathrm{~g} / \mathrm{cm}^{3}$.

Os valores médios de absorção de água variaram de 7,34\% a 48,18\% após 2 horas, e de $22,52 \%$ a $85,18 \%$ após 24 horas de imersão em água. Tanto para 2 , quanto para 24 horas de imersão, os painéis de Pinus taeda, apresentaram valor médio de absorção de água estatisticamente superior em relação aos painéis produzidos com madeiras tropicais. Para 2 horas de absorção, os painéis de Eschweilera coriácea apresentaram média estatisticamente inferior em relação aos painéis das demais espécies. Já, para 24 horas de absorção, os painéis de Eschweilera coriácea e Manilkara amaz̧ônica foram os que apresentaram média estatisticamente inferior em relação aos painéis das demais espécies.

Os valores médios de inchamento em espessura variaram de $2,05 \%$ a $15,49 \%$ após 2 horas, e de 6,89\% a $35,23 \%$ após 24 horas de imersão em água. Tanto para 2 , quanto para 24 horas de imersão, os painéis de Pinus taeda, apresentaram valores médios de inchamento em espessura estatisticamente superiores em relação aos demais tratamentos. Para inchamento após 2 horas, os painéis de Eschweilera coriácea, Manilkara amazônica, Eschweilera odora e
Pouteria guianensis apresentaram média estatisticamente inferior em relação aos painéis das demais espécies. Para inchamento após 24 horas, os painéis de Manilkara amazônica e Eschweilera coriacea apresentaram média estatisticamente inferior aos painéis das demais espécies.

\section{Propriedades mecânicas dos painéis}

$\mathrm{Na}$ tabela 4 estão apresentados os valores médios de módulo de ruptura, módulo de elasticidade, tração perpendicular, resistência ao arrancamento de parafusos na superfície e topo dos painéis.

Os valores médios de módulo de ruptura (MOR) variaram de 10,01 MPa (Swartzia recurva) a 16,95 MPa (Pinus taeda). Os painéis produzidos com madeira de Eschweilera coriacea e Eschweilera odora apresentaram médias estatisticamente iguais aos painéis de Pinus taeda, espécie empregada como base referencial. Entre as seis espécies tropicais, os painéis de Eschweilera coriácea, Eschweilera odora, Pouteria guianensis e Byrsonima crispa apresentaram média estatisticamente iguais entre si e superiores em relação aos painéis de Manilkara amarônica e Swartzia recurva. 
TABELA 4. Resultados médios das propriedades mecânicas dos painéis.

\begin{tabular}{lccccc}
\hline Tratamento & $\begin{array}{c}M O R \\
(\mathrm{MPa})\end{array}$ & $\begin{array}{c}\text { MOE } \\
(\mathrm{MPa})\end{array}$ & $\begin{array}{c}T P \\
(\mathrm{MPa})\end{array}$ & $\begin{array}{c}\text { RAP-S } \\
(\mathrm{N})\end{array}$ & $\begin{array}{c}\text { RAP-T } \\
(\mathrm{N})\end{array}$ \\
\hline Byrsonima crispa & $14 \mathrm{bc}$ & $2151 \mathrm{~b}$ & $1,01 \mathrm{a}$ & $1310 \mathrm{ab}$ & $1470 \mathrm{ab}$ \\
& $(16,79)$ & $(12,54)$ & $(16,77)$ & $(6,03)$ & $(24,13)$ \\
Eschweilera coriacea & $15 \mathrm{ab}$ & $2692 \mathrm{a}$ & $0,80 \mathrm{~b}$ & $1288 \mathrm{ab}$ & $1179 \mathrm{~b}$ \\
& $(13,99)$ & $(9,62)$ & $(19,90)$ & $(4,11)$ & $(13,50)$ \\
Eschweilera odora & $15 \mathrm{ab}$ & $2119 \mathrm{~b}$ & $1,04 \mathrm{a}$ & $1237 \mathrm{~b}$ & $1491 \mathrm{a}$ \\
& $(16,49)$ & $(14,59)$ & $(15,00)$ & $(5,96)$ & $(22,59)$ \\
Manilkara amazonica & $11 \mathrm{c}$ & $2050 \mathrm{~b}$ & $0,75 \mathrm{bc}$ & $1175 \mathrm{~b}$ & $1094 \mathrm{~b}$ \\
& $(24,52)$ & $(15,05)$ & $(24,43)$ & $(7,04)$ & $(29,82)$ \\
Pouteria guianensis & $14 \mathrm{~b}$ & $2206 \mathrm{~b}$ & $0,83 \mathrm{~b}$ & $1444 \mathrm{a}$ & $1183 \mathrm{~b}$ \\
& $(16,86)$ & $(13,73)$ & $(18,15)$ & $(8,13)$ & $(25,22)$ \\
Swartzia recurva & $10 \mathrm{c}$ & $1719 \mathrm{c}$ & $0,83 \mathrm{~b}$ & $1172 \mathrm{~b}$ & $1205 \mathrm{~b}$ \\
& $(19,85)$ & $(17,47)$ & $(15,98)$ & $(7,29)$ & $(24,69)$ \\
Pinus taeda & $16 \mathrm{a}$ & $2192 \mathrm{~b}$ & $0,70 \mathrm{c}$ & $1395 \mathrm{a}$ & $1580 \mathrm{a}$ \\
& $(21,53)$ & $(9,95)$ & $(14,85)$ & $(4,34)$ & $(11,95)$ \\
\hline
\end{tabular}

MOR: Módulo de ruptura; MOE: Módulo de elasticidade; TP: Tração perpendicular ao plano do painel; RAP-S/RAP-T: Resistência ao arranchamento de parafuso - superfície/topo; Médias seguidas da mesma letra na mesma coluna são estatisticamente iguais pelo teste de Tukey a 95\% de probabilidade. Valores entre parêntese indicam o coeficiente de variação. Valores ajustados por análise de covariância para massas específica aparentes de $0,731 \mathrm{~g} / \mathrm{cm}^{3}$ (Flexão estática), $0,750 \mathrm{~g} / \mathrm{cm}^{3}$ (Tração perpendicular), 0,722 g/ $\mathrm{cm}^{3}$ (RAP).

Para o módulo de elasticidade (MOE), os valores médios variaram de $1719 \mathrm{MPa}$ (Swartzia recurva) a $2692 \mathrm{MPa}$ (Eschweilera coriácea). Os painéis de Eschweilera coriácea apresentaram média estatisticamente superior em relação às demais espécies, inclusive à espécie testemunha (Pinus taeda). Com exceção de Swartz̨ia recurva, os painéis de Byrsonima crispa, Echweilera odora, Manilkara amazônica e Pouteria guianensisi apresentaram médias estatisticamente iguais em relação à espécie testemunha.

Os valores médios de tração perpendicular variaram de 0,70 $\mathrm{MPa}$ (Pinus taeda) a 1,01 $\mathrm{MPa}$ (Eschweilera odora). Os painéis produzidos com madeiras de Eschweilera odora e Byrsonima crispa apresentaram médias estatisticamente superiores em relação às demais espécies. A menor média de tração perpendicular foi obtida para os painéis de Pinus taeda, sendo estatisticamente inferior em relação às cinco das seis espécies de madeiras tropicais estudadas.

Os valores médios de resistência ao arrancamento de parafusos variaram de $1172 \mathrm{~N}$ (Swartzia recurva) a $1395 \mathrm{~N}$ (Pinus taeda) para a superfície, e de 1094 N (Manilkara amazônica) a $1580 \mathrm{~N}$ (Pinus taeda) para o topo dos painéis.
Para a RAP na superfície, os painéis de Byrsonima crispa, Eschweilera coriacea e Pouteria guianensis apresentaram médias estatisticamente iguais aos painéis de Pinus taeda, espécie empregada como base referencial. Para a RAP no topo, os painéis de Byrsonima crispa e Eschweilera odora apresentaram médias estatisticamente iguais a Pinus taeda. Entre as espécies tropicais, com exceção de Eschweilera odora, todas as demais espécies apresentaram médias de RAP no topo estatisticamente iguais entre si.

\section{DISCUSSÕES}

Os valores médios de massa específica básica obtidos para as seis espécies de madeiras tropicais foram bem superiores ao valor médio obtido para o Pinus taeda.

Os resultados para teor de extrativos totais obtidos para todas as espécies estudadas estão abaixo do limite máximo de 10\%, conforme citado por Moslemi (1974). Como referência, Prata (2010) obteve para Pinus taeda valor de extrativos totais de 3,48\%; Iwakiri et al. (2015) encontraram para duas espécies de madeiras tropicais os 
seguintes valores de extrativos totais: Inga alba (4,61\%) e Swartzia recurva (6,53\%).

Todos os valores de $\mathrm{pH}$ obtidos para as espécies estudadas estão dentro da faixa de 3,0 a 6,0 conforme relatado por Moslemi (1974). A madeira de Manilkara amaẑnonica apresentou $\mathrm{pH}$ abaixo do valor obtido para Pinus taeda de 4,58. Como referência, Prata (2010) obteve para Pinus taeda, valor de pH de 4,7; Iwakiri et al. (2015) encontraram para duas espécies de madeiras tropicais, Inga alba e Swartzia recurva, valores de $\mathrm{pH}$ de 5,30 e 5,24 respectivamente.

Com exceção de Pinus taeda, empregada como espécie testemunha, todos os painéis produzidos com as seis espécies de madeiras tropicais apresentaram razão de compactação abaixo de 1.3, valor referenciado como mínimo por Moslemi (1974).

A menor massa específica da madeira de Pinus taeda, e seu efeito sobre o aumento da razão de compactação dos painéis, não contribuiu para a redução da absorção de água. Vital et al. (1974) analisando os efeitos do aumento na razão de compactação dos painéis aglomerados de 1,2 para 1.6, constataram uma redução na absorção de água, e atribuíram a este aumento a maior compactação das partículas de madeira e consequente redução na porosidade, dificultando a entrada de água na estrutura mais fechada dos painéis. Os painéis produzidos com as seis espécies de madeiras tropicais com menor massa específica e maior razão de compactação apresentaram menor absorção de água. Para fins de comparações com os resultados desta pesquisa, Trianoski, Piccardi, Iwakiri, Matos e Bonduelle (2016) encontraram para painéis produzidos com mistura de Pinus taeda com Grevilea robusta, valores de $22,44 \%$ a $31,41 \%$ para AA 24 h. Portanto, os resultados de absorção 24 horas obtidos para as espécies tropicais podem ser considerados satisfatórios.

O inchamento em espessura é uma propriedade que define a estabilidade dimensional dos painéis aglomerados. Nesta pesquisa, constatou-se que os painéis de Pinus taeda, com maior razão de compactação, apresentaram inchamento em espessura superior em relação aos painéis produzidos com espécies tropicais com maior densidade da madeira e menor razão de compactação. Moslemi (1974) afirma que painéis com maior razão de compactação apresentam maior inchamento em espessura, devido à maior liberação das tensões de compressão resultantes da prensagem, além do inchamento higroscópico da maior quantidade de partículas de madeira.

Os resultados de inchamento em espessura obtidos nesta pesquisa estão compatíveis em relação a alguns valores apresentados na literatura. Trianoski, Iwakiri, Matos e Chies (2013), Iwakiri et al. (2014) e Trianoski et al. (2016), encontraram para painéis produzidos com pinus, valores médios de inchamento em espessura 24 horas de 18,61\%, $24,22 \%$ e $31,41 \%$, respectivamente.

Tanto para o MOR, quanto para o MOE, não foram constatadas influências significativas da densidade da madeira e da razão de compactação sobre os resultados destas propriedades, indicando a possibilidade de uso de espécies de maior densidade na produção de painéis aglomerados.

Com exceção dos painéis de Swartria recurva e Manilkara amazonica, todas as demais espécies apresentaram médias de MOR superiores ao requisito mínimo de $13 \mathrm{MPa}$, conforme estabelecido pela Norma EN 312:2003 (EN, 2003). Já, para o MOE, todos os tratamentos propostos apresentaram médias superiores ao requisito mínimo de $1600 \mathrm{MPa}$, estabelecido pela referida Norma.

Os valores médios de MOR e MOE obtidos neste trabalho foram satisfatórios em comparação a alguns resultados apresentados na literatura para painéis produzidos com madeira de pinus. Para o MOR os valores referenciados foram de 11,17 $\mathrm{MPa}$ (Trianoski, Iwakiri e Matos 2011), 13,94 MPa (Trianoski et al. 2016) e 12,78 MPa (Iwakiri et al. 2014). Já para o MOE, os valores foram de $1581 \mathrm{MPa}$ (Trianoski et al. 2011), $1751 \mathrm{MPa}$ (Trianoski et al. 2016) e $1847 \mathrm{MPa}$ (Iwakiri et al. 2014).

A maior razão de compactação dos painéis de Pinus taeda não contribuiu para o aumento nos resultados de tração perpendicular, conforme relatado na literatura. Da mesma forma, os painéis produzidos com as seis espécies tropicais com razão de compactação abaixo do mínimo recomendado de 1,3 apresentaram resultados satisfatórios 
para esta propriedade. Os resultados obtidos para todos os tratamentos propostos foram superiores ao requisito mínimo de 0,35 $\mathrm{MPa}$ estabelecido pela Norma EN 312:2003 (EN, 2003).

Com relação aos resultados de pesquisas apresentados na literatura, Colli et al. (2010), encontraram para painéis aglomerados produzidos com Schizolobium amaz̧onicum valor médio de tração perpendicular de 0,22 MPa. Já, Vital et al. (1974) encontraram para painéis aglomerados de Virola spp., com razão de compactação de 1,2 : 1.0 (baixa) e 1,6 : 1.0 (alta), valores de tração perpendicular de $0,48 \mathrm{MPa}$ e 0,65 MPa, respectivamente. Para painéis de pinus, Trianoski et al. (2011), Trianoski et al. (2016) e Iwakiri et al. (2014), encontraram valores de tração perpendicular de 1,05 MPa; 1,14 MPa e 0,58 MPa, respectivamente.

Quanto à resistência ao arrancamento de parafusos, todos os tratamentos atingiram os requisitos mínimos estabelecidos pela Norma NBR 14810-2:2006 (ANBT, 2006a) de $1020 \mathrm{~N}$ para a superfície e de $800 \mathrm{~N}$ para o topo dos painéis.

Os resultados obtidos neste trabalho estão próximos aos encontrados por Bianche (2009), para painéis produzidos com madeiras de Eucalyptus urophylla, Schizolobium amazonicum e Sida spp., cujos valores variaram de $1027 \mathrm{~N}$ a $1376 \mathrm{~N}$ (topo e superfície). Já para painéis produzidos com madeira de pinus, Trianoski et al. (2016) encontraram valores de RAP-S e RAP-T de 1137 N e 1042 $\mathrm{N}$, respectivamente.

\section{CONCLUSÕES}

As seis espécies tropicais avaliadas nesta pesquisa apresentaram razão de compactação dos painéis abaixo do mínimo de 1,3 devido a maior massa específica de suas madeiras. Entretanto, esta relação não afetou de forma significativa os resultados das propriedades físicas e mecânicas dos painéis.

Com base nas avaliações comparativas dos resultados desta pesquisa com os requisitos da norma EN 312 (2003) (EN, 2003) e valores obtidos para os painéis de Pinus taeda, utilizada como espécie referencial, pode-se afirmar que, as espécies Byrsonima crispa, Eschweilera coriacea, Eschweilera odora e Pouteria guianensis apresentam grande potencial para produção de painéis aglomerados.

\section{AGRADECIMENTOS}

Os autores registram seus agradecimentos ao INCT/INPA/CNPq - Instituto Nacional de Ciência e Tecnologia / Madeiras da Amazônia, pela concessão das madeiras utilizadas nesta pesquisa e ao apoio logístico e financeiro para a coleta e transporte do material até o local de estudo. À empresa BERNCEK Painéis e Serrados S.A., pela doação de partículas de pinus e adesivo utilizado nesta pesquisa.

\section{REFERÊNCIAS}

Associação Brasileira de Normas Técnicas [ABNT] (2006a). NBR 14810 - 2 - Chapas de madeira aglomerada - Parte 2 Requisitos. São Paulo: ABNT.

Associação Brasileira de Normas Técnicas [ABNT] (2006b). NBR 14810 - 3 - Chapas de madeira aglomerada - Parte 3 Métodos de Ensaios. São Paulo: ABNT

Bianche, J. J. (2009). Propriedades de aglomerados fabricados com particulas de Eucalipto (Eucalyptus urophylla), Paricá (Schizolobium amazonicum) e Vassoura (Sida spp.). Dissertação (Mestrado em Ciências Florestais), Universidade Federal de Viçosa. Viçosa.

Comissão Panamericana de Normas Técnicas [COPANT] (1972). COPANT 461. Determinación del peso especifico aparente. Lima; COPANT.

European Standards [ES] (2002a). EN 310. Determination of modulus of elasticity in bending and bending strength. Brussels: ES.

European Standards [ES] (2002b). EN 317. Determination of swelling in thickness after immersion in water. Brussels: ES.

European Standards [ES] (2002c). EN-319. Determinação da resistência à tração perpendicular às faces da placa. Lisboa: ES.

European Standards [ES] (2002d). EN 323. Determinação da massa volúmica. Versão Portuguesa. Lisboa: ES.

European Standards [ES] (2003). EN 312. Particleboards Specifications. Brussels: ES.

Colli, A., Vital, B. R., Carneiro, A. C. O., Silva, J. C., Carvalho A., N. M. L., \& Della Lucia, R. M. (2010). Propriedades de painéis produzidos com partículas de madeira de Paricá (Schizolonium amazonicum Huber ex.Ducke) e fibras de coco. Árvore, 34(2), 333-338. 
Gonçalves, F. G., Oliveira, J., Lucia, R., \& Sartório, R. (2009). Estudo de algumas propriedades mecânicas da madeira de híbrido clonal de Eucalyptus urophylla X Eucalyptus grandis. Árvore, 33(2), 501-509.

Iwakiri, S., Latorraca, J. V. F., Silva, D. A; Gabardo, J. L., Klitzke, R. J., Fofano, A., Fabrowski, F., \& Interanmense, M. T. (1996). Produção de chapas de Madeira aglomerada de Pinus elliottii (Engelm) and Eucalyptus dunnii (Maid). Agrárias, 15(2), 33-41.

Iwakiri, S., Vianez, B. F., Weber, C., Trianoski, R., \& Almeida, V. C. (2012). Avaliação das propriedades de painéis aglomerados produzidos com resíduos de serrarias de nove espécies de madeiras tropicais da Amazônia. Acta Amazônica, 42(1), 59-64.

Iwakiri, S., Trianoski, R., Cunha, A. B., Castro, V. G., Braz, R. L., Villas Boas, B. T., Sanches, F. L., \& Bellon, K. R. R. (2014). Evaluation of the quality of particleboard panels manufactured with wood from Sequoia sempervirens and Pinus taeda. Cerne, 20, 209-216.

Iwakiri, S., Trianoski, R., Nascimento, C. C., Gumane, C., Lengowski, E. C., Schardosin, F. Z., \& Azambuja, R. (2015). Resistência das juntas coladas de madeiras de Inga alba (SW) Wild e swartiia recurva Poep. Cerne, 21(3), 457-463.

Kelly, M. W. A. (1977). Critical literature review of relationships between processing parameters and physical properties of particleboards. Madison, Winscosin: U.S. Forest Products Laboratory.

Maloney, T. M. (1993). Modern particleboard and dry-process fiberboard manufacturing (2 ed.). São Francisco: Miller Freeman

Marra, A. A. (1992). Technology of Wood bonding: principles and practice. New York: Van Nostrand Reinhold.

Moslemi, A. A. (1974). Particleboard. London: Southern Illinois University Press.

Prata, J.G. (2012). Estudo da viabilidade tecnológica do uso de espécies de pinus tropicais para produção de painéis colados lateralmente - EGP. Dissertação (Mestrado em Ciências Florestais), Universidade Federal do Paraná. Curitiba.

Technical Association of the Pulp and Paper Industry [TAPPI] (1997). TAPPI 204. Solvent extractives of wood and pulp. Atlanta: TAPPI.
Technical Association of the Pulp and Paper Industry [TAPPI] (2002). TAPPI 252. $p H$ and electrical conductivity of hot water extracts of pulp, paper, and paperboard. Atlanta: TAPPI.

Trianoski, R., Iwakiri, S., \& Matos, J. L. M. (2011). Potential use of planted fast-growing species for production of particleboard. Journal of Tropical Forest Science, 23, 311-317.

Trianoski, R., Iwakiri, S., Matos, J. L. M., \& Chies, D. (2013). Utilização da madeira de Cryptomeria japonica para produção de painéis aglomerados. Scientia Forestalis, 41, 029-064.

Trianoski, R., Piccardi, A. B. R., Iwakiri, S., Matos, J. L. M., \& Bonduelle, G. M. (2016). Incorporação de Grevilea robusta na produção de painéis aglomerados de Pinus spp. Floresta \& Ambiente, 23, 278-285. doi: 10.1590/2179-8087.141515

Tsoumis, G. (1991). Science and technology of wood: structure, properties and utilization. New York: Van Nostrand Reinhold.

Vital, B. R., Lehmann, W. F., \& Boone, R. S. (1974). How species and board densities affect properties of exotic hardwood particleboards. Forest Products Journal, 24(12), 37-45.

O manuscrito recebeu a 19 de janeiro de 2017

Aceite a 23 de abril de 2018

Postou a 18 de dezembro de 2018

\section{Este documento deve ser citado como:}

Iwakiri, S., Trianoski, R., do Nascimento, C. C., Juizo, C. G. F., Lengowski, E. C., Bilcati, G. K., \& Gonçalves. (2018). T.Painéis aglomerados produzidos com seis espécies de madeiras tropicais da Amazônia. Madera y Bosques, 24(3), e243371. doi: $10.21829 /$ myb.2018.243371

Madera y Bosques por Instituto de Ecología, A.C. se distribuye bajo una Licencia Creative Commons Atribución-NoComercialCompartirlgual 4.0 Internacional. 